\title{
Demo: Ground Temperature Difference Driven Sensor for Environmental Monitoring
}

\begin{tabular}{|c|c|}
\hline Natsuki Ikeda & Junichiro Shiomi \\
\hline The University of Tokyo & The University of Tokyo \\
\hline 7-3-1 Hongo, Bunkyo-ku, Tokyo, & 7-3-1 Hongo, Bunkyo-ku, Tokyo, \\
\hline JAPAN & JAPAN \\
\hline ikeda@akg.t.u-tokyo.ac.jp & shiomi@photon.t.u-tokyo.ac.jp \\
\hline Ryo Shigeta & Yoshihiro Kawahara \\
\hline The University of Tokyo & The University of Tokyo \\
\hline 7-3-1 Hongo, Bunkyo-ku, Tokyo, & 7-3-1 Hongo, Bunkyo-ku, Tokyo, \\
\hline JAPAN & JAPAN \\
\hline shigeta@akg.t.u-tokyo.ac.jp & kawahara@akg.t.u-tokyo.ac.jp \\
\hline
\end{tabular}

Permission to make digital or hard copies of part or all of this work for personal or classroom use is granted without fee provided that copies are not made or distributed for profit or commercial advantage and that copies bear this notice and the full citation on the first page. Copyrights for third-party components of this work must be honored. For all other uses, contact the owner/author(s).

Copyright held by the owner/author(s)

UbiComp/ISWC'18 Adjunct, October 8-12, 2018, Singapore, Singapore ACM 978-1-4503-5966-5/18/10.

https://doi.org/10.1145/3267305.3267575

\begin{abstract}
In this paper, we present a novel approach to the realization of a battery-free soil profile probe that uses the temperature difference between the near-surface air and the underground soil as a power source. The temperature change in the underground soil is slower than that in the near-surface air, and thus a large temperature difference occurs between the near-surface air and the underground soil for most of the day. Hence, we developed a sensor prototype driven by a thermoelectric generator (TEG) that directly converts this temperature difference into electricity. The results of an experimental implementation of the prototype proved that when the difference in temperature between the nearsurface air and the underground soil is only $3^{\circ} \mathrm{C}$, which is much lower than the average temperature difference in an actual field, the measured output power is about $80 \mu \mathrm{W}$. Because the typical sensing interval of a soil profile probe is $1 \mathrm{~h}$, the average power consumption (e.g., for a Texas Instruments CC2650) is about $5 \mu \mathrm{W}$, which is much lower than the expected amount of harvested energy.
\end{abstract}

\section{Author Keywords}

Energy Harvesting; Thermoelectric Generator

\section{ACM Classification Keywords}

B.0. Hardware: General 

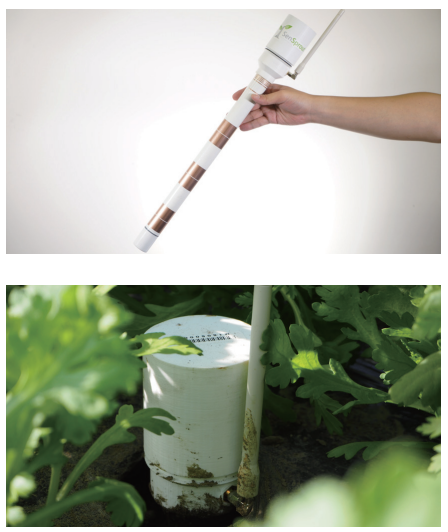

Figure 1: (Upper image) SenSprout battery-driven agricultural soil profile probe for monitoring soil moisture and temperatures at multiple depths of $(10 \mathrm{~cm}, 20 \mathrm{~cm}, 30 \mathrm{~cm})$, as well as the ground surface temperature. (Lower image) Agricultural soil profile probe covered with vegetation.

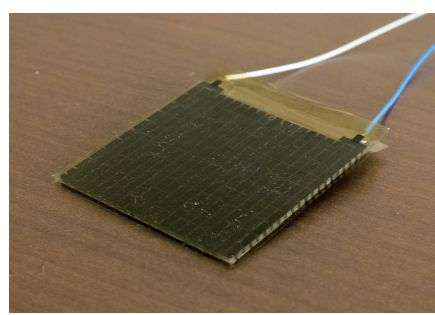

Figure 2: Thermoelectric generator KELK KTGM199-2.

\section{Introduction}

A profile probe (Figure 1 (Upper image)) monitors the distribution of the soil moisture and temperature at different underground depths, which is valuable for irrigation management and the other agricultural operations. Because many sensors are deployed in farm fields, manually replacing the batteries is impractical when considering the labor cost. Taking the soil profile probe that we developed as an example [8], the theoretical battery life is limited to around 1 year despite a sensing interval of only $1 \mathrm{~h}$. When operating in a severe environment such as under hot and humid conditions, the battery life is limited to only a few months because of battery deterioration. Therefore, battery-free sensors are preferred for reducing the maintenance costs. Moreover, because the sensors are installed near the ground surface, they will inevitably become covered with vegetation (Figure 1 (Lower image)). Solar panels [5] are not suitable for such scenarios, because the panels need to compete with the vegetation to acquire sunlight. The temperature change in the underground soil is slower than in the nearsurface air, and thus a large temperature difference occurs between the near-surface air and the underground soil for most of the day (Figure 3) [7]. Hence, we developed a sensor prototype driven by a thermoelectric generator (TEG) and showed the variability of our proposal through an experimental implementation.

\section{Thermoelectric generator (TEG)}

TEG is a solid-state device (Figure 2) that converts the heat flux (temperature difference) directly into electrical energy through a phenomenon called the Seebeck effect. The Seebeck coefficient, also known as thermoelectric power, of a device is a measure of the magnitude of the thermoelectric voltage induced in response to the temperature difference across the device, as occurring through the Seebeck effect. The SI unit of the Seebeck coefficient is volts per kelvin.
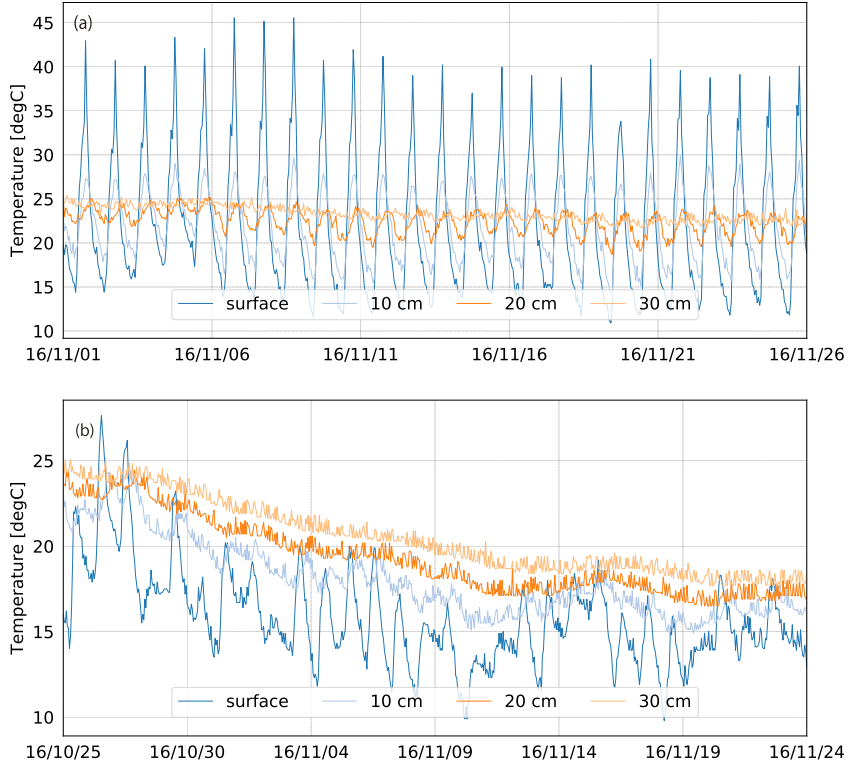

Figure 3: Near-surface air and underground soil temperature data from an actual field ((a) Omitama, Ibaraki, Japan / (b) Benoda, India).

Using the Seebeck coefficient $(\alpha[\mathrm{V} / \mathrm{K}])$ and the temperature difference $(\Delta T[\mathrm{~K}])$, the induced thermoelectric voltage $\left(V_{\mathrm{TEG}}[\mathrm{V}]\right)$ can be explained as follows:

$$
V_{\mathrm{TEG}}=\alpha \times \Delta T
$$

A TEG functions similarly to a heat engine, but it differs in that it has no moving parts and has a high fault tolerance. Many types of studies on a TEG have been conducted in different fields [1, 6, 9]. For example, Gou et. al. showed the viability and further performance capability of a TEG for waste heat recovery in the industrial area [1]. Leonov 


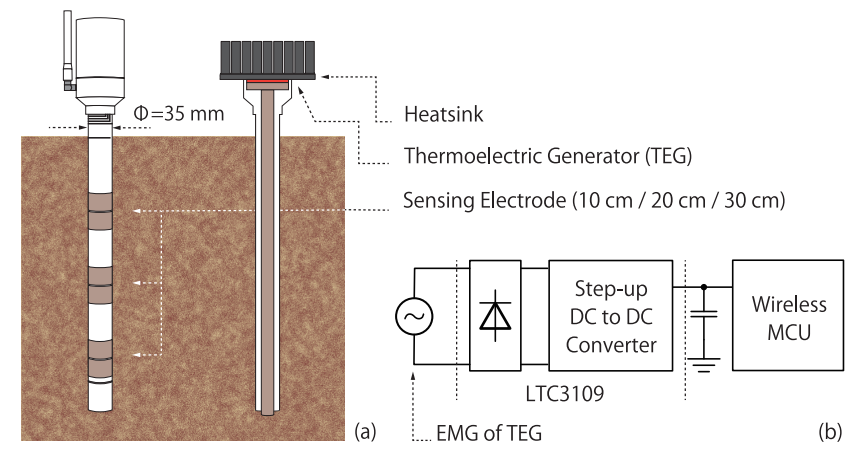

Figure 4: (a) Current soil profile probe (left side) and cross-sectional view of our proposed battery-free sensor (right side). (b) Schematic of the electric circuit.

Table 1: Characteristics of the TEG.

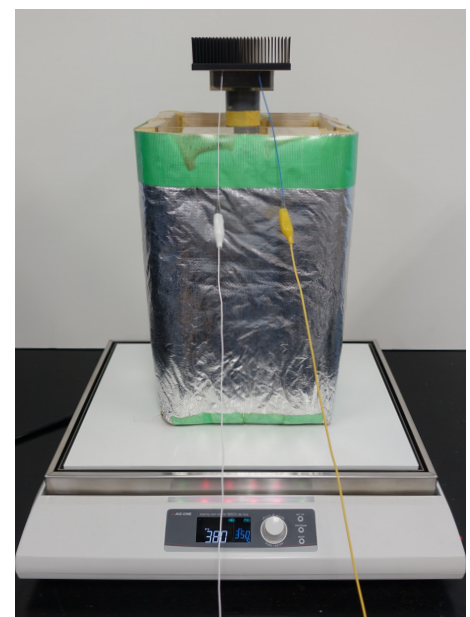

Figure 5: Experimental environment simulating typical situations in farm fields. proposed system, the temperature of the underground soil $(30 \mathrm{~cm})$ is guided toward the lower side of TEG (KELK KTGM199-2 [4]) using a copper rod wrapped with a thermal insulator. Table 1 shows the characteristics of the TEG. A heat sink (ALPHA LC100-30B [3]) is attached to the upper side of the TEG to accelerate the heat exchange with the near-surface air. Because the differences in temperature between the near-surface air and the underground soil constantly fluctuate (Figure 3), the TEG output voltage changes dynamically, and the electric current direction switches daily. A power manager for the TEG (Analog Devices LTC3109 [2]) manages this variability using autopolarity, step-up DC-to-DC conversion, and energy storage (Figure 4 (b)). When the output voltage of LTC3109 $\left(V_{\text {OUT }}[\mathrm{V}]\right)$ is $3.3 \mathrm{~V}$, the relationship between the TEG voltage $\left(V_{\text {TEG }}[\mathrm{mV}]\right)$ and the output current of the LTC3109 $\left(I_{\text {OUT }}[\mu \mathrm{A}]\right)$ is as described below [2]:

$$
I_{\mathrm{OUT}} \simeq \begin{cases}0 & \left(\left|V_{\mathrm{TEG}}\right| \leq 30 \mathrm{mV}\right) \\ 3 \times V_{\mathrm{TEG}}-90 \frac{[\mu \mathrm{A}]}{[\mathrm{mV}]} & \left(\left|V_{\mathrm{TEG}}\right| \geq 30 \mathrm{mV}\right)\end{cases}
$$

\section{Demonstration Experiment}

We prepared an experimental environment simulating typical situations in farm fields. As shown in Figure 5, we arranged a box surrounded by a thermal insulator and filled it with soil. We heated the bottom of the box with an experimental hot plate to reproduce the field environment where the temperature gradient occurs in the vertical direction of the soil. When the difference in temperature between the near-surface air and the underground $(30 \mathrm{~cm})$ is $3^{\circ} \mathrm{C}$, the output power of LTC3109 is $80 \mu \mathrm{W}$. Note that this difference in temperature is much lower than the average temperature difference in the field. Considering an ultra-low power MCU (e.g., Texas Instruments CC2650), the power consumption in standby-mode is $2.1 \mu \mathrm{W}$, and the power consumption for 
each sensing and data-transmission is $100 \mu \mathrm{J}$. Because a typical sensing span of a soil profile probe is $1 \mathrm{~h}$, the average power supply required is approximately $5 \mu \mathrm{W}$, which is much lower than the expected harvested energy. Thus, we realized battery-free soil profile probes driven by the difference in temperature between the near-surface air and the underground soil.

\section{Conclusion}

In this paper, we showed the feasibility of taking advantage of the temperature difference between between the nearsurface air and the underground soil, and proved through an experimental implementation that soil profile probes can be driven using a TEG. In the future, we will conduct a demonstration in a real field to validate the utility of our proposal.

\section{Acknowledgements}

I would like to thank Union-Farm (Omitama, Ibaraki, Japan) and Professor J. Adinarayana (Indian Institute of Technology Bombay) for providing data used in this research. This work was supported by JST ERATO, Japan under Grant No.: JPMJER1501.

\section{REFERENCES}

1. Xiaolong Gou, Heng Xiao, and Suwen Yang. 2010. Modeling, Experimental Study and Optimization on Low-Temperature Waste Heat Thermoelectric Generator System. Appl. Energy. 87, 10 (Oct. 2010), 3131-3136. DOI:http://dx.doi.org/10.1016/j. apenergy . 2010.02.013

2. Analog Devices Inc. 2018. LTC3109-Auto-Polarity, Ultralow Voltage Step-Up Converter and Power Manager. (May 2018). Retrieved May 21, 2018 from http://www.analog.com/media/en/technicaldocumentation/data-sheets/3109fb.pdf
3. Alpha Company Ltd. 2018a. LC100. (May 2018). Retrieved May 21, 2018 from https://www . micforg. co.jp/dxf/LC.pdf

4. KELK Ltd. 2018b. Thermoelectric Module Data. (May 2018). Retrieved May 21, 2018 from https: //www. kelk.co.jp/generation/index.html

5. Raghunathan Vijay, Kansal Aman, Hsu Jason, Friedman Jonathan, and Srivastava Mani. 2005 Design Considerations for Solar Energy Harvesting Wireless Embedded Systems. In Proceedings of the 4th International Symposium on Information Processing in Sensor Networks (IPSN '05). IEEE, 457-462. DOI : http://dx.doi.org/10.1109/IPSN . 2005.1440973

6. Leonov Vladimir, Torfs Tom, Fiorini Paolo, and Hoof Chris Van. 2007. Thermoelectric Converters of Human Warmth for Self-Powered Wireless Sensor Nodes. IEEE Sens. J. 7, 5 (May 2007), 650-657. DOI : http: // $\mathrm{dx}$. doi .org/10.1109/JSEN. 2007.894917

7. Jury William A and Horton Robert. 2004. Soil physics. John Wiley \& Sons, Inc., New York, NY, USA.

8. Shirahama Yasutomo, Shigeta Ryo, Kawahara Yoshihiro, and Asami Tohru. 2015. Implementation of Wide Range Soil Moisture Profile Probe by Coplanar Plate Capacitor on Film Substrate. In Proceedings of IEEE SENSORS 2015 (IEEE Sens '15). IEEE, 1-4. DOI : http://dx.doi.org/10.1109/ICSENS. 2015. 7370633

9. Tan Yen Kheng and Panda Sanjib Kumar. 2011. Energy Harvesting From Hybrid Indoor Ambient Light and Thermal Energy Sources for Enhanced Performance of Wireless Sensor Nodes. IEEE on Trans. Ind. Electron. 58, 9 (Sept. 2011), 4424-4435. DOI : http://dx.doi . org/10.1109/TIE. 2010.2102321 